\title{
Expansion transversale de la crête alvéolaire mandibulaire en deux temps. Illustration par un cas clinique
}

\section{RÉSUMÉ}

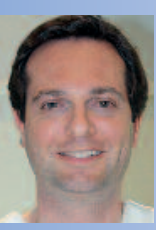

Nicolas VINAY

Service d'Odontologie, CHRU de Montpellier,

549 avenue du Pr J.-L. Viala, 34295 Montpellier cedex 5.

Marie-Alix FAUROUX

Service d'Odontologie, CHRU de Montpellier.

Jacques-Henri TORRES

Service d'Odontologie, CHRU de Montpellier.
La gestion de l'insuffisance de volume osseux dans les secteurs postérieurs mandibulaires est un problème courant en implantologie. Plusieurs techniques ont été décrites afin d'augmenter le volume osseux alvéolaire. Ces techniques incluent les greffes de blocs osseux en " onlay ", la régénération osseuse guidée, la distraction ostéogénique et l'expansion transversale de crête par clivage sagittal. Dans cette dernière technique, le fragment osseux vestibulaire est luxé latéralement après fracture en bois vert, et l'espace ainsi créé entre les corticales vestibulaire et linguale est comblé par de l'os autogène, un substitut osseux ou simplement rempli par le caillot sanguin. L'expansion transversale de crête est habituellement réalisée dans le même temps opératoire que la pose des implants.

Cette technique est plus adaptée au maxillaire qu'à la mandibule de par la qualité osseuse moins dense permettant une certaine flexibilité. À la mandibule, le risque de malfracture du fragment osseux vestibulaire est grand du fait d'une plus grande rigidité due à l'épaisseur des corticales. Une approche en deux étapes peut être envisagée afin de réduire le risque de malfracture et de nécrose osseuse.

Cet article présente un cas clinique d'expansion transversale modifié, en deux temps opératoires : trois semaines après la corticotomie d'un segment osseux rectangulaire vestibulaire, la crête alvéolaire a été clivée sagittalement en laissant le périoste relié au segment vestibulaire latéralisé, et deux implants ont été mis en place. 


\section{Introduction}

$>$

La gestion de l'insuffisance de volume osseux dans les secteurs latéraux mandibulaires est un problème courant en implantologie. Devant des crêtes alvéolaires minces, d'une largeur inférieure à 4 ou $5 \mathrm{~mm}$, il faut procéder à une augmentation osseuse afin de pouvoir placer des implants selon les techniques classiques, en ménageant idéalement un rempart osseux d'au moins $1 \mathrm{~mm}$ de part et d'autre de l'implant. Diverses solutions existent pour augmenter le volume osseux : la régénération osseuse guidée (ROG), les greffes osseuses d'apposition latérale, la distraction ostéogénique, et l'expansion osseuse transversale par ostéotomie communément appelée "bone-splitting » ou « ridge-splitting » [1-3].

Cette dernière méthode, introduite par Simion M. et al. [4] en 1992, consiste à créer un nouveau lit osseux implantaire élargi après ostéotomie sagittale le long de la crête alvéolaire. Depuis quelques années, la technique a considérablement bénéficié du développement des instruments piézoélectriques [5]. Leur coupe très fine et très précise et leur non-agressivité vis-à-vis des tissus mous contribuent à augmenter le taux de succès de ces interventions et permettraient de réduire la perte osseuse marginale péri-implantaire [6].

Il est intéressant de comparer l'expansion osseuse transversale par ostéotomie aux techniques les plus fréquemment utilisées pour élargir une crête osseuse édentée [7-8]. On reproche souvent à la ROG son coût de mise en œuvre et le risque de complications infectieuses, notamment en cas d'exposition de la membrane. De plus, tout comme les greffes osseuses, la ROG nécessite une période de cicatrisation d'au minimum 5 à 6 mois selon le matériau utilisé. Ces matériaux, qu'il s'agisse d'os autogène broyé, d'os allogène ou de xénogreffes, nécessitent également une rétention mécanique à l'aide de membranes renforcées dont l'utilisation constitue une difficulté technique opératoire supplémentaire et un risque supplémentaire d'échec, a fortiori lorsqu'il s'agit de reconstructions de grande étendue. Les greffes de blocs osseux en « onlay » ostéosynthésés, contrairement à la ROG, ne nécessitent pas de protection particulière en dehors du recouvrement par les tissus mous, mais présentent d'autres inconvénients tels que la morbidité lié aux sites donneurs intra ou extra-oraux, ainsi que les risques de résorption du greffon, qui varieraient de 20 à $50 \%$ après six mois [9]. L'expansion ou l'augmentation transversale par ostéotomie constitue donc en comparaison une alternative [10] relativement simple qui permet dans de nombreux cas d'éviter des greffes osseuses aux résultats variables [11-14]. L'expansion osseuse transversale apparaît donc supérieure aux autres procédures d'augmentation osseuse, en termes de taux de succès, de coût et de morbidité.

Au cours d'une intervention d'expansion osseuse transversale, le fragment osseux cortical vestibulaire est luxé latéralement après une fracture en « bois vert ». L'espace créé entre le mur osseux lingual et le fragment cortical vestibulaire ainsi déplacé peut être comblé à l'aide d'un matériau de substitution osseuse, d'os autogène, ou simplement rempli par le caillot sanguin. Au maxillaire, l'expansion osseuse transversale est aisée. Elle est basée sur la plasticité de l'os, et la mobilisation des fragments osseux peut être obtenue simplement, à l'aide d'expanseurs de crêtes [15]. Le pronostic est statistiquement excellent. À la mandibule, en 
revanche, le risque de malfracture du segment osseux est élevé du fait de la grande rigidité que confère à cet os une corticale beaucoup plus épaisse que celle du maxillaire.

De ce fait, l'ostéotomie longitudinale de la crête mandibulaire doit impérativement être complétée par des ostéotomies verticales de " décharge ".

Malgré ces traits verticaux, la fracture basale du segment osseux vestibulaire n'est pas contrôlable, et un trait basal est encore nécessaire. Alors qu'au maxillaire, où la crête conserve sa vascularisation basale après expansion, à la mandibule, le fragment mobilisé est privé de l'ensemble de ses connexions vasculaires, ce qui
I'expose théoriquement à un risque de résorption, voire de nécrose : on se retrouve dans le même cas de figure que pour une greffe d'apposition non vascularisée [11-12].

Par ailleurs, si les implants placés au maxillaire dans un os expansé montrent un taux de succès à cinq ans compris entre 86 et $99 \%$ selon Sethi A. et al. [7], les implants placés à la mandibule dans les mêmes conditions auraient un taux d'échec plus élevé [4].

Ces constatations ont amené Enislidis G. et al. [11] à développer pour la mandibule une technique d'expansion osseuse transversale modifiée [11-13], dite en deux temps ou "par étapes" (fig. 1). Cette méthode cherche à obtenir une
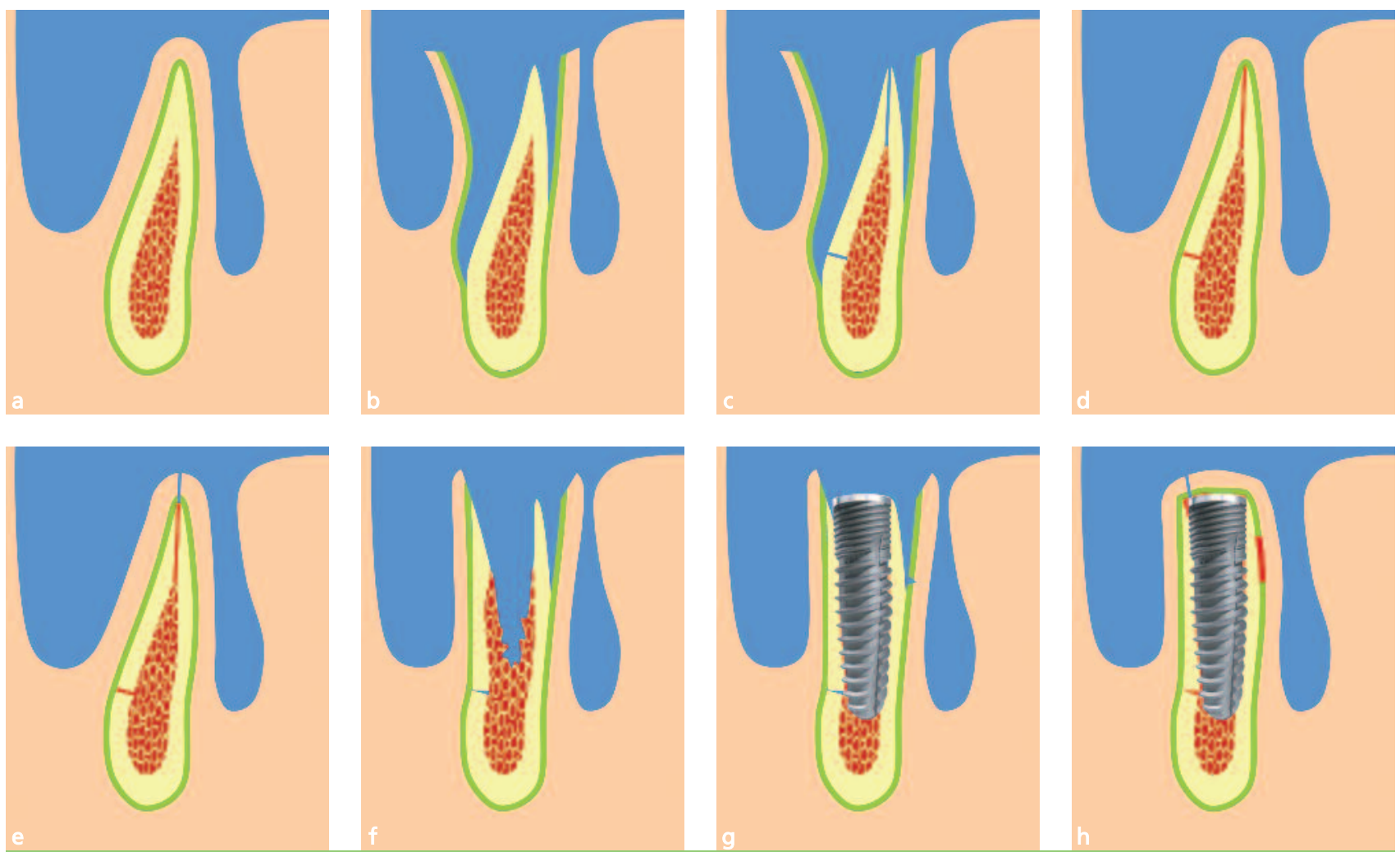

Fig. 1 a à $h$ Technique de l'expansion transversale en deux temps.

Premier temps : la crête fine (a) est exposée (b) puis la corticale est découpée au Piézotome ${ }^{\circledR}$ (c) ; les lambeaux sont suturés (d). Deuxième temps : trois à quatre semaines plus tard, la muqueuse est incisée en regard de la découpe (e); seul un lambeau lingual est élevé et le volet prédécoupé est luxé du côté vestibulaire (f) ; les implants sont mis en place (g) et le périoste est incisé pour donner de la laxité au lambeau lingual et permettre la fermeture du site $(h)$. 
fracture osseuse bien contrôlée par une prédécoupe réalisée dans une première intervention. Lors de la seconde intervention, ce fragment osseux vestibulaire est luxé et déplacé sans décollement de lambeau, dans le but de préserver l'apport vasculaire d'origine périostée. Le respect de la vascularisation du volet osseux rapproche cette technique des greffes pédiculées. Enislidis G. et al. [11] affirment dans leur conclusion que cette technique peut être considérée comme fiable et qu'elle permet d'éviter les problèmes rencontrés avec une technique en 1 temps sans augmenter significativement le temps de traitement.

Plus récemment dans leur publication de 2010, Sohn DS. et al. [13] ont comparé la technique en 2 temps avec celle en 1 temps. Leur étude a porté sur 32 patients ayant une crête mandibulaire postérieure de 2 à $4 \mathrm{~mm}$ d'épaisseur. Sur les 32 patients, 23 ont été traités selon une technique d'expansion latérale en 1 temps (immé-

\section{Cas clinique}

Une patiente de 45 ans, sans pathologie particulière, présentait un édentement postérieur terminal mandibulaire droit. Un traitement implantaire a été envisagé pour remplacer 45 et 46 . L'examen clinique a révélé une crête très mince, et l'imagerie (fig. 2) a confirmé que le volume osseux était insuffisant pour placer des implants selon la technique classique. Il a été opté pour une expansion de la crête alvéolaire en deux temps.

$\mathrm{Au}$ cours d'une première intervention, un volet osseux cortical vestibulaire a été isolé par un trait " haut " d'ostéotomie piézoélectrique (Piézotome 2, Satelec ${ }^{\circledR}$ ) qui a fendu la crête sagittalement, deux traits verticaux « de décharge ", et un trait horizontal « bas » reliant diate) et 9 selon une technique d'expansion latérale en 2 temps (retardée). Sur les 23 patients ayant été traités en 1 temps, 5 ont présenté une malfracture du volet osseux vestibulaire ayant nécessité l'utilisation de vis d'ostéosynthèse pour stabiliser celui-ci en bonne position ou modifier la position idéale des implants. En revanche, sur les 9 patients ayant subi la technique en 2 temps, la fracture du volet osseux vestibulaire s'est faite conformément au trait de corticotomie apical préréalisé et laissant le périoste attaché. Après quatre à cinq mois, tous les implants étaient ostéointégrés selon les critères de Buser D. et al. [16], sauf chez un patient qui avait bénéficié de la technique immédiate. Dans son cas, le fragment osseux s'est résorbé, laissant apparaître un séquestre. Les auteurs ont conclu que la technique en 2 temps (retardée) devait être recommandée chez les patients présentant une crête mandibulaire très fine ou dans les cas de forte densité osseuse et de corticale épaisse.

les incisions verticales et délimitant un rectangle (fig. 3). Ce volet n'a pas été luxé ; le lambeau a été simplement suturé.

$\mathrm{Au}$ cours d'une seconde intervention à $\mathrm{J}+21$ jours, une incision muqueuse crestale isolée (sans incision de décharge et surtout sans décollement de lambeau vestibulaire), a été pratiquée au-dessus du trait d'ostéotomie crestal réalisé trois semaines auparavant (fig. 4 a). Le segment cortical "prédécoupé " lors de la première intervention a été luxé à l'aide d'une lame malléable coudée à $90^{\circ}$ introduite dans le trait d'ostéotomie crestal, que l'on a fait agir en rotation pour écarter les deux pièces osseuses. Les implants ( $3 \mathrm{i}$, diamètre $=3,5 \mathrm{~mm}$ longueur $=10 \mathrm{~mm}$ ) ont été insérés après un 


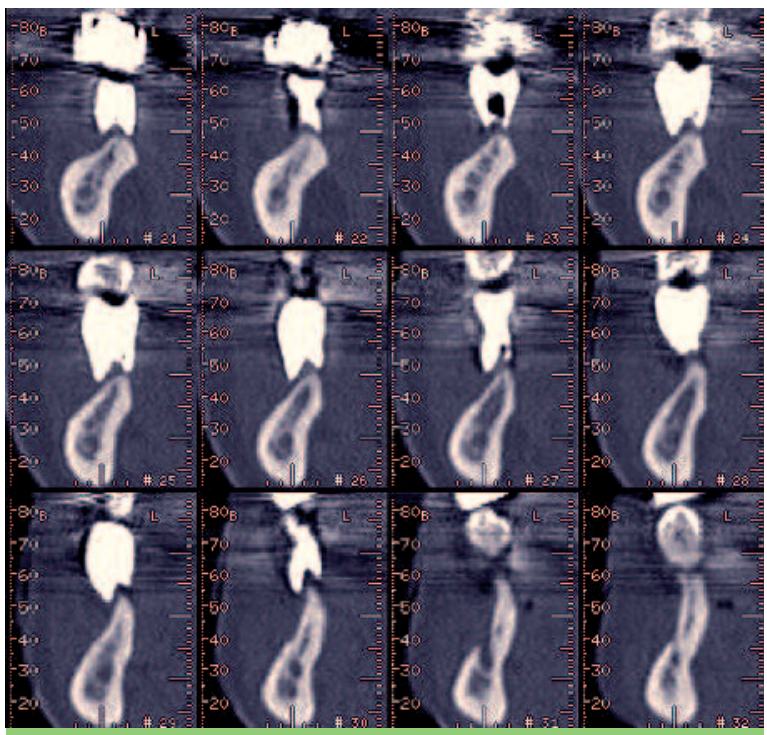

Fig. 2 Aspect initial de la crête mandibulaire édentée en tomodensitométrie (" scanner »).

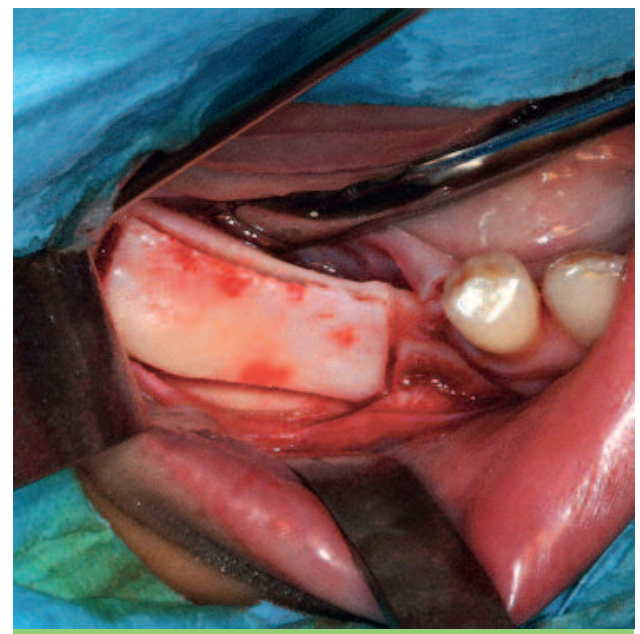

Fig. 3 Découpe du volet lors de la première intervention. léger forage destiné à préparer la partie apicale des puits. Une vis d'ostéosynthèse a été utilisée pour stabiliser fermement le volet vestibulaire à la corticale linguale (fig. 4 b). Elle a été mise en place directement à travers la muqueuse et sa tête est restée apparente à la fin de la chirurgie. Cette vis a aussi participé à la stabilité primaire des implants. Aucun biomatériau de substitution osseuse n'a été mis en place. Le lambeau lingual a été libéré pour permettre une fermeture muqueuse sans tension (fig. 4 c). Dans les jours qui ont suivi, la tête de la vis d'ostéosynthèse a été recouverte par la muqueuse.
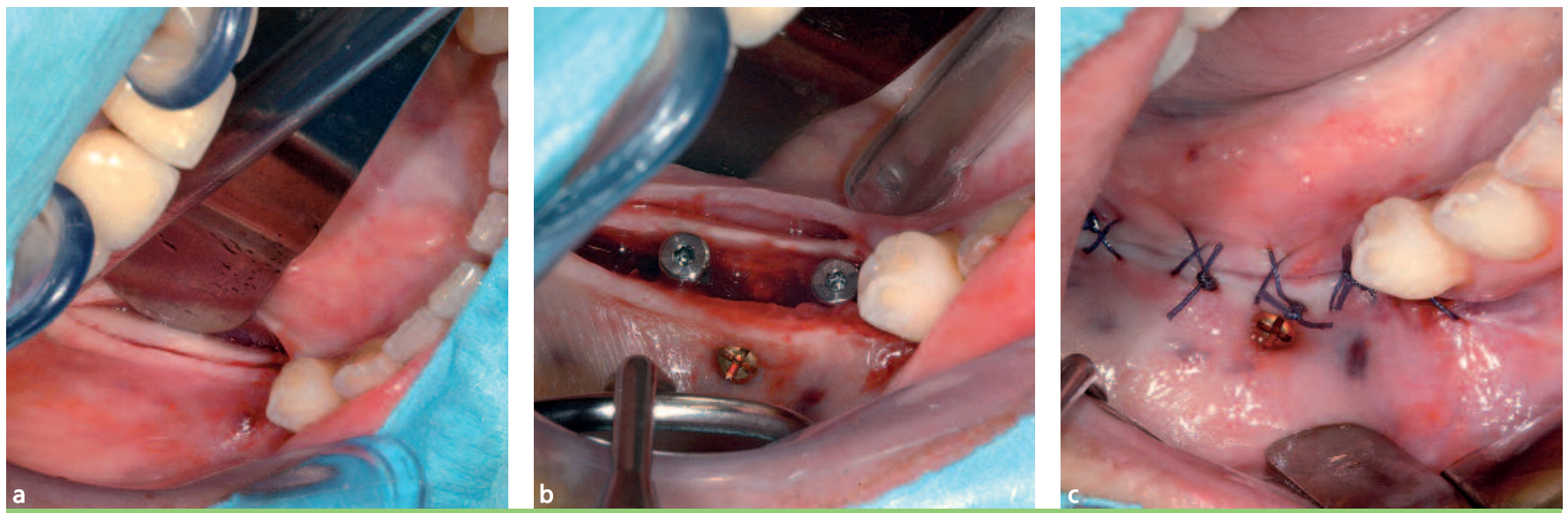

Fig. 4 a à c Seconde intervention.

(a) : la muqueuse est incisée et un lambeau lingual élevé.

(b) : après luxation du volet vestibulaire, les implants sont posés et une vis d'ostéosynthèse stabilise le segment osseux.

(c) : le lambeau lingual est suturé. 

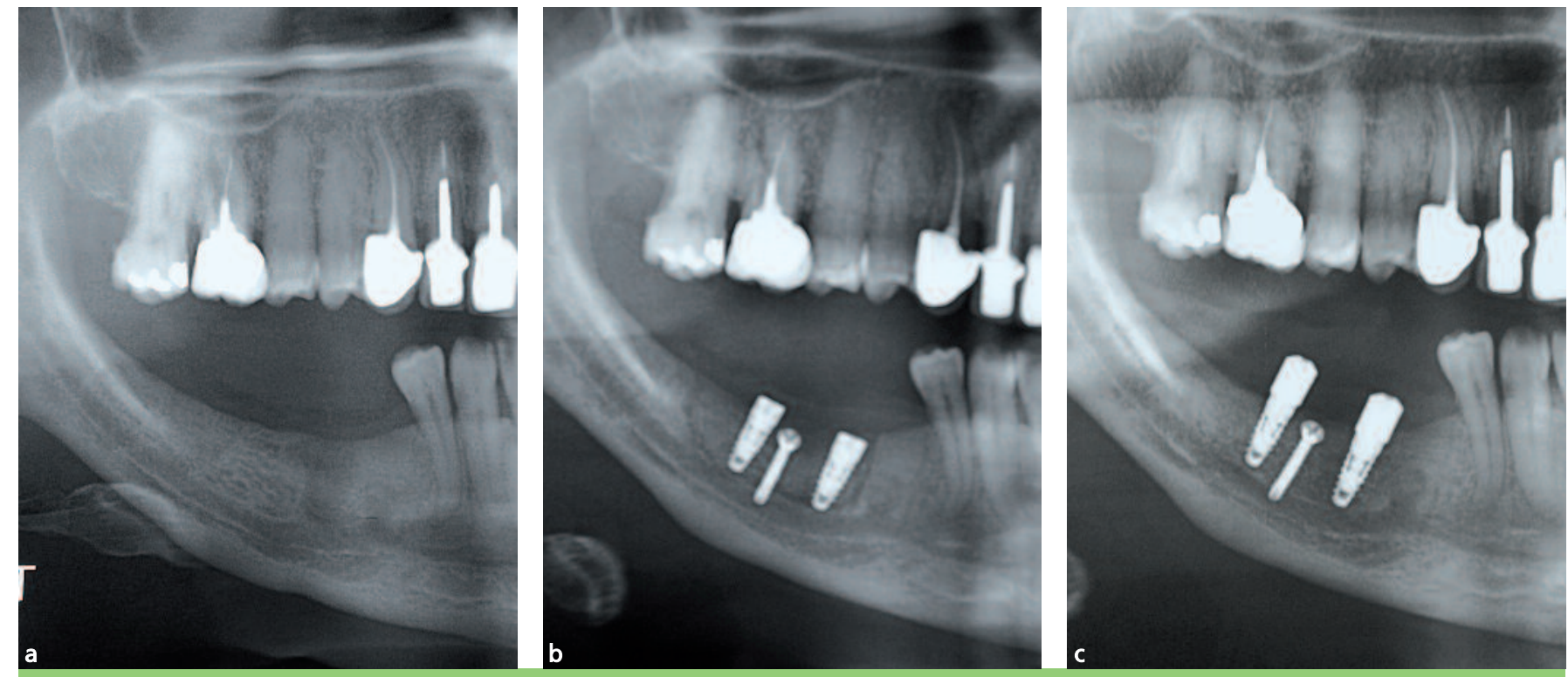

Fig. 5 a à c Orthopantomogrammes.

(a) : initial

(b) : 7 jours après la première intervention, le contour du volet fracturé est nettement visible. La pointe de la vis d'ostéosynthèse se projette sur l'image du canal mandibulaire mais elle est plus linguale que lui.

(c) : 5 mois après la prmière intervention, le contour du volet n'est plus discernable. Les vis de cicatrisation des implants sont en place.
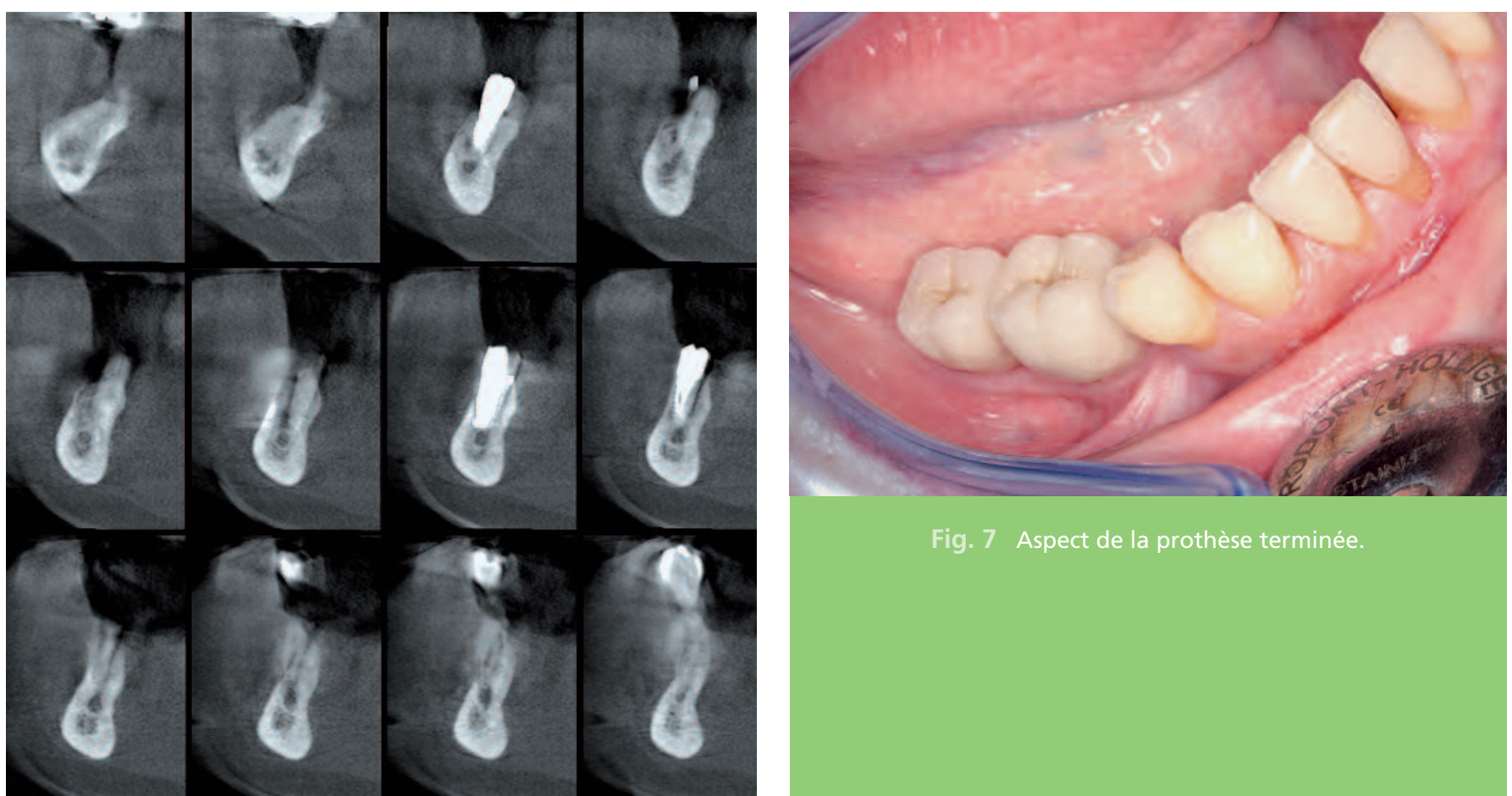

Fig. 7 Aspect de la prothèse terminée.

Fig. 6 Tomographie volumique à faisceau conique (« cone beam ») à six mois. 
À cinq mois, les implants ont été mis en fonction, et la vis d'ostéosynthèse déposée. La cicatrisation des tissus péri-implantaires apparaissait tout à fait satisfaisante. Les contrôles radiographiques pratiqués montraient la consolidation du volet fracturé (fig. 5).

Afin de mieux appréhender le résultat, une imagerie sectionnelle a été réalisée (fig. 6). II semblerait que la corticale vestibulaire se soit

\section{Conclusion}

L'intérêt de ce protocole d'expansion de crête en deux temps réside dans le respect de la vascularisation du volet osseux lors de sa luxation, ce qui contribue à réduire les complications liées à la trophicité (exposition, infection, résorption) souvent observées dans les greffes d'apposition à la mandibule.

Les résultats bibliographiques de la technique en deux temps illustrée par ce cas clinique mon- en partie résorbée, mais la hauteur d'os a été conservée et la crête a effectivement été élargie par rapport à la situation de départ. D'un point de vue clinique, les implants étaient parfaitement stables et la phase de réalisation prothétique s'est ensuite déroulée classiquement.

Après 12 mois de recul, la patiente est tout à fait satisfaite du résultat fonctionnel et esthétique (fig. 7). trent que cette intervention est facile à réaliser, prédictible et sûre.

Par rapport à la chirurgie en 1 temps, elle améliore le résultat sans allonger significativement le temps de traitement.

Elle devrait donc être indiquée en première intention dans les cas d'insuffisance transversale sévère de volume osseux dans les secteurs postérieurs mandibulaires.

\section{Remerciements}

Réalisation prothétique : Laboratoire DT, 22 rue Agenais, 34080 Montpellier. Imagerie sectionnelle : Dr Y. Brédoire, 10 rue de la Loge, 34000 Montpellier.

\section{Bibliographie}

1. Coatoam GW, Mariotti A. The segmental ridge-split procedure.

J Periodontol 2003;74(5):757-70.

2. Misch $\mathrm{CM}$. Implant site development using ridge splitting techniques.

Oral Maxillofac Surg

Clin North Am

2004;16(1):65-74, vi.

3. Elo JA, Herford AS,

Boyne PJ.
Implant success

in distracted bone

versus autogenous bone-grafted sites.

J Oral Implantol 2009;35(4):181-4.

4. Simion $\mathrm{M}$, Baldoni $\mathrm{M}$, 
Zaffe D.

Jawbone enlargement using immediate implant placement associated with a split-crest technique and guided tissue regeneration. Int J Periodontics Restorative Dent 1992;12(6):462-73.

5. Peivandi $A$, Bugnet $R$, Debize E, Gleizal A, Dohan DM.

[Piezoelectric osteotomy: applications in periodontal and implant surgery].

Rev Stomatol Chir

Maxillofac

2007;108(5):431-40.

6. Danza M, Guidi R, Carinci F. Comparison between implants inserted into piezo split and unsplit alveolar crests. J Oral Maxillofac Surg 2009;67(11):2460-5.

7. Sethi A, Kaus T. Maxillary ridge expansion with simultaneous implant placement: 5-year results of an ongoing clinical study. Int J Oral Maxillofac Implants 2000;15(4):491-9.

8. Aghaloo TL, Moy PK. Which hard tissue augmentation techniques are the most successful in furnishing bony support for implant placement?
Int J Oral Maxillofac

Implants 2007;22 Suppl:49-70.

9. Cordaro L, Amadé DS, Cordaro M.

Clinical results

of alveolar ridge

augmentation with

mandibular block bone

grafts in partially

edentulous patients prior

to implant placement.

Clin Oral Implants Res

2002;13(1):103-11.

10. Basa S, Varol A, Turker N. Alternative bone expansion technique for immediate placement of implants in the edentulous posterior mandibular ridge:

a clinical report.

Int J Oral Maxillofac

Implants 2004;19(4):554-8.

11. Enislidis G, Wittwer G,

Ewers R.

Preliminary report

on a staged ridge splitting

technique for implant

placement in the mandible:

a technical note.

Int J Oral Maxillofac

Implants 2006;21(3):445-9.

12. González-García R, Monje F, Moreno C.

Alveolar split osteotomy

for the treatment

of the severe narrow ridge

maxillary atrophy:

a modified technique.

Int J Oral Maxillofac Surg
2011;40(1):57-64.

13. Sohn DS, Lee HJ, Heo JU, Moon JW, Park IS,

Romanos GE.

Immediate and delayed lateral ridge expansion technique in the atrophic posterior mandibular ridge. J Oral Maxillofac Surg 2010;68(9):2283-90.

14. Piccinini $M$.

Mandibular bone expansion technique in conjunction with root form implants: a case report. J Oral Maxillofac Surg 2009;67(9):1931-6.

15. Lalo J, Chassignolle V, Beleh M, Djemil M.

[Maxillary ridge expansion for dental implant placement with alveolar corticotomy].

Rev Stomatol Chir Maxillofac 2008;109(5):316-22.

16. Buser $D$, Mericske-Stern $R$, Bernard JP, Behneke A, Behneke N, Hirt HP, Belser UC, Lang NP. Long-term evaluation of non-submerged ITI implants.

Part 1: 8-year life table analysis of a prospective multi-center study with 2359 implants. Clin Oral Implants Res 1997;8(3):161-72. 


\section{SUMMARY}

\section{Two stage lateral mandibular ridge splitting. Illustration through a clinical case}

Nicolas VINAY,

Marie-Alix FAUROUX, Jacques-Henri TORRES

\section{Keywords}

ridge splitting

dental implant
The management of the atrophic posterior mandibular ridge is a common problem in implant dentistry.

Numerous surgical techniques have been described to increase the ridge volume. These strategies include onlay block bone grafting, guided bone regeneration, distraction osteogenesis or ridge split/ridge expansion technique.

The lateral ridge expansion technique aims to create a new implant bed by longitudinal osteotomy of the alveolar bone. The buccal cortex is repositioned laterally, after a greenstick fracture, and the space between the buccal and lingual cortical plates is filled with autologous, allogenic or alloplastic graft material or left without any graft material. The lateral ridge expansion technique is usually performed simultaneously with implant placement.

This technique is more suitable to the maxilla than the mandible owing to the thinner cortical plates and softer medullary bone. In the mandible, the risk of malfracture of the osteotomized buccal segment is greater because of lower flexibility and thicker cortical plates, and a staged approach can also be used to avoid malfracture and bone necrosis in the mandible.

The present report shows a clinical case of a modified staged approach in the mandible: after corticotomy of a rectangular buccal segment and a 3 weeks healing, the mandibular ridge was split, leaving the periostum attached to the lateralized segment, and 2 implants were placed.

\section{NOWELLE REVUE}

\section{Droit et Médecine Bucco-Dentaire La revue de I'actualité en droit dentaire}

Directeur de la rédaction : Alain Béry

Rédacteurs en chef : Laurent Delprat et Charles Georget

Il y a plusieurs années, sous l'impulsion d'André Demichel, est apparue l'émergence du droit médical. Désormais, devant la spécialisation croissante des techniques et du droit, l'instauration d'un droit dentaire spécifique semble relever de l'évidence.

C'est dans cet esprit de reconnaissance que l'Association de Droit Dentaire, portée par l'indéfectible soutien de sa Présidente d'honneur Francine Demichel, a souhaité procéder à la création de cette nouvelle revue de "Droit et Médecine Bucco-Dentaire".

La publication, trimestrielle, permet de rendre compte rapidement de l'actualité juridique, qu'il s'agisse de nouvelles lois ou jurisprudences, réformes, études de cas, ou autres décisions touchant votre exercice quotidien.

$$
\text { Abonnez-vous en ligne! }
$$

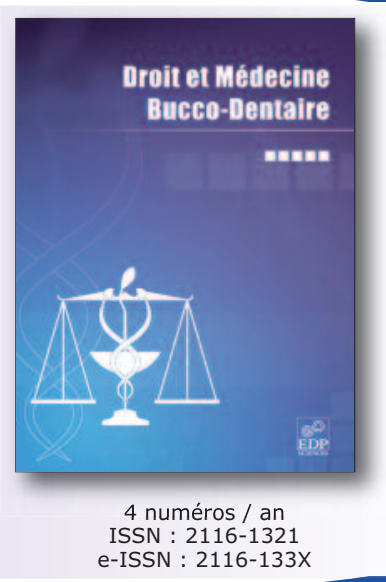

\section{WwW.rdmbd.org}

